\title{
Covid-19 Vaccination: A Bird's Eye View
}

\author{
Tahir Sultan Shamsi1 ${ }^{1, *}$, Mehjabeen Imam² \\ ${ }^{1}$ Department of Clinical Hematology, National Institute of Blood Disease \& Bone Marrow Transplantation, Karachi, Pakistan. \\ ${ }^{2}$ Department of Immunology, National Institute of Blood Disease \& Bone Marrow Transplantation, Karachi, Pakistan.
}

Covid-19 pandemic plagued this world since the beginning of 2020 AD. It is caused by a new positive-strand RNA virus of coronaviridae family [1]. It causes Coronavirus disease 2019 (hence the name COVID-19). It is a contagious disease predominantly causes severe acute respiratory syndrome, hence the name SARS-CoV-2. It started from Wuhan, China, in December 2019. Since then, it has spread globally.

It is reported to be a new virus therefore it's properties, pathogenesis, virulence, immunogenicity, variants, and how will host body will react to this virus was unknown. Despite of 22 months since this virus started to spread worldwide, researchers and clinicians continued to learn about it on daily basis. Newer information about it poured in daily in scientific journals as well as in print / electronic media. Mostly, newer information continued to negate earlier information. Social media disinformation continued to confuse the masses.

Being a new virus, no diagnostic kit, specific drug or vaccine was available. As of now, various diagnostic kits and vaccines developed in different countries and many got regulatory approval under "Emergency Use Authorization" on fast track [2]. Hundreds of drugs to combat Covid-19 virus are under development and undergoing clinical trials.

Covid-19 vaccines developed using different platforms and are grouped according to the technology used in its development. Vaccines that got entry in phase 3 trials include: mRNA vaccines (Pfizer, BioNTech, Moderna), replication-defective viral vector vaccines (Astra-Zeneca, Sputnik-V, Johnson \& Johnson), inactivated pathogen vaccines (Sinopharm, Sinovac), protein subunit vaccines (Novavax), and virus-like vaccines (GSK) [3]. In Pakistan, Sinopharm and Sinovac were provided to the masses supported by WHO Covax program. Inactivated viral vaccines contain whole pathogen that has been treated with heat or chemical so that it losses the pathogenic antigens. Their immunogenicity is enhanced by using alum as adjuvant and giving booster shots after 4 to 8 weeks of the first shot [4]. In the category of nucleic acid vaccines, Pfizer (mRNA vaccine) is the prototype which is the first ever FDA approved mRNA vaccine. It is based on mRNA molecule that contains genetic expression for spike protein encapsulated in lipid nanoparticle vector. mRNA is translated by the host to produce potent viral antigens that will produce

*Address correspondence to this author at the Department of Clinical Hematology, National Institute of Blood Disease \& Bone Marrow Transplantation, Karachi, Pakistan. Email: t.shamsi.62@gmail.com adaptive immune response directed against $\mathrm{S}$ protein of virus [5].

Usually, anticipated time from developing a vaccine and to be available for clinical use takes a decade or more. In index case, development, and availability of vaccine for clinical use within a year of onset is commendable on one hand. But its long-term safety and efficacy couldn't be assessed through the same stringent procedure as for routine vaccines in use. Social media disinformation about vaccine created chaos in layman population that resulted in reluctance in their mind to accept it as a "safe" preventive strategy. Imbalance between supply and demand of the vaccine has been another significant issue especially in third world countries. Mass production of vaccine was started in developed countries shortly after the pandemic started. Therefore, their population got vaccinated long before rest of the world. Luckily, in most countries, general population didn't get to pay for the cost of vaccine. It was supplied through Covax program of WHO or respective governments made sure that there shouldn't be cost implications for public.

Regarding safety of different available vaccines, different local and systemic complications have been reported worldwide for all the vaccines. However, no deaths among vaccine recipients have been reported directly attributable to it. Usual vaccine related issues of pain at injection site, swelling, fever or chills are the commoner symptoms post injection. Vaccine induced thrombosis or thrombocytopenia have been extremely rare complication. Other late effects involving different organ systems also have been brought up anecdotally.

As far as efficacy is concerned, protection from re-infection is reported in more than $90 \%$ vaccinated people. According to $\mathrm{CDC}$, vaccine provides two-fold more protection than natural infection alone. Those who developed Covid-19 post vaccination had less morbidity as compared to non-vaccinated patients [6].

Another observation reported is the decline of COVID-19 antibodies in subsequent months (4-6 months) after vaccine. This phenomenon was also noted after COVID-19 infection in most patients. Those who had severe infection developed higher of antibodies while those with moderate symptoms or asymptomatic infection, developed lower titer. According to a study published in nature by Washington University School of Medicine in St. Louis, vaccination induced strong antibody 
responses especially in people who had been previously exposed to COVID-19 infection then those who had never been infected. Their germinal centers were found to be active after four months of vaccine's first dose [7].

There is a lot of debate about the need of booster doses of vaccine. High risk individuals have started getting it in some countries. Whether it will become a standard practice or not remains to be decided. Nature has published an article in May 2021 in which many studies suggest that mixing different vaccines provokes persuasive immune responses as compared to the use of only single vaccine. However, the major concern regarding this mix-and-match theory is the emergence of side effects and to identify the ideal time for giving the booster shot. At least 12 weeks spacing is preferred before giving the booster [8].

Almost two years on, we still don't know much about COVID-19. There are lots of unanswered questions. Till such time, we all must follow experts working in this area in different countries. Gossip and here-say should not be followed. It is hoped we will see a control of this pandemic in near future. Till that day, we should follow all preventive measures and get vaccinated.

\section{CONFLICT OF INTEREST}

Declared none.

\section{ACKNOWLEDGEMENTS}

Declared none.

\section{REFERENCES}

[1] Ortiz-Prado E, Simbaña-Rivera K, Gómez-Barreno, L, et al. Clinical, molecular and epidemiological characterization of the SARS-CoV-2 virus and the Coronavirus disease 2019 (COVID-19), a comprehensive literature review. Diagn Microbiol Infect Dis 2020; 98(1): 115094. DOI: 10.1016/j.diagmicrobio.2020.115094

[2] US Food and Drug Administration. Emergency Use Authorizations for Medical Devices. 2021; Available at: https:/www.fda.gov/medical-devices/emergency-situations-medical-devices/emergency-use-authorizations-medical-devices.

[3] Kyriakidis NC, López-Cortés A, González EV, Grimaldos AB, Prado EO. SARS-CoV-2 vaccines strategies: A comprehensive review of phase 3 candidates. Vaccines $2021 ; 6(1)$ : 1-7. DOI: 10.1038/s41541-021-00292-w

[4] Gao Q, Bao L, Mao H, et al. Development of an inactivated vaccine candidate for SARS-CoV-2. Science 2020; 369(6499): 77-81. DOI: 10.1126/science.abc1932

[5] Jackson LA, Anderson EJ, Rouphael NG, et al. An mRNA Vaccine against SARS-CoV-2-preliminary report. N Engl J Med 2020; 383: 1920-31. DOI: 10.1056/NEJMoa2022483

[6] Media Statement. New CDC Study: Vaccination offers higher protection than previous COVID-19 infection. 2021; Available at: https://www.cdc.gov/media/releases/2021/s0806-vaccination-protection.html

[7] Bhandari T. COVID-19 vaccine generates immune structures critical for lasting immunity. USA: Washington University School of Medicine. 2021; Available at: https://medicine.wustl.edu/news/covid-19-vaccine-generates-immune-structures-critical-for-lasting-immunity

[8] Callaway E. Mixing COVID vaccines trigger potent immune response. Nature 2021; 593: 491. DOI: $10.1038 / \mathrm{d} 41586-021-01359-3$ 